\title{
Can we develop effective combination antiangiogenic therapy for patients with hepatocellular carcinoma?
}

\author{
Justin B. Wenger $\cdot$ Napoleon Santos $\cdot$ Yanxia Liu $\cdot$ Jennifer Dallas $\cdot$ \\ Sukanthini Subbiah $\cdot$ Steven Hochwald $\cdot$ Emina H. Huang $\cdot$ Duyen T. Dang • \\ Carmen J. Allegra $\cdot$ Hendrik Luesch $\cdot$ Long H. Dang
}

Received: 9 April 2011/ Accepted: 18 July 2011/Published online: 9 August 2011

(C) The Author(s) 2011. This article is published with open access at Springerlink.com

\begin{abstract}
Antiangiogenic therapy has shown promise in the treatment of patients with hepatocellular carcinoma (HCC). Bevacizumab, sorafenib, and sunitinib showed efficacy in patients with HCC; and sorafenib is approved by the FDA for treatment of this cancer. In practice, the clinical benefit of these agents has been heterogeneous; and in patients who do respond, the benefit is modest and/or short-lived. Recent advances in the molecular understanding of tumor angiogenesis along with the rapid development of targeted drug discovery have made it possible to explore novel combination therapy for HCC. We review the clinical trial results, discuss possible molecular
\end{abstract}

J. B. Wenger and N. Santos contributed equally to this work.

J. B. Wenger - N. Santos - J. Dallas · S. Subbiah .

C. J. Allegra - L. H. Dang $(\varangle)$

Division of Hematology/Oncology,

Department of Internal Medicine,

University of Florida Shands Cancer Center,

University of Florida, 1600 SW Archer Road,

Gainesville, FL 32610, USA

e-mail: long.dang@medicine.ufl.edu

Y. Liu · H. Luesch $(\bowtie)$

Department of Medicinal Chemistry,

University of Florida, 1600 SW Archer Road,

Gainesville, FL 32610, USA

e-mail: luesch@cop.ufl.edu

S. Hochwald · E. H. Huang

Department of Surgery, University of Florida,

Gainesville, USA

D. T. Dang

Department of Internal Medicine,

University of Michigan, Ann Arbor, MI, USA mechanisms of resistance, and suggest novel combinations with antiangiogenic therapy.

Keywords HIF- $1 \alpha \cdot$ HIF- $2 \alpha$ - Antiangiogenic therapy · Hepatocellular carcinoma

\section{Introduction}

Liver cancer is the fifth most common malignancy and the third most common cause of cancer-related mortality worldwide [1]. Even though more than $80 \%$ of the cases occur in developing countries, its incidence continues to increase in developed countries largely due to the Hepatitis $\mathrm{C}$ epidemic [2]. Hepatocellular carcinoma (HCC) represents the major histologic subtype and accounts for an estimated $70-85 \%$ of the cases [3]. Because of the asymptomatic nature of early $\mathrm{HCC}$, most patients present with unresectable disease [4]. Presently, curative rates for patients with advanced disease are dismal due to the resistant nature of this malignancy to systemic chemotherapy. Unfortunately, local interventions such as chemoembolization and ablation have failed to significantly extend life expectancy [5]. And even in those who undergo surgical resection, intrahepatic recurrence occurs in $70 \%$ of the patients [6].

Recently, antiangiogenic therapy has shown promise in the treatment of patients with HCC. This novel class of agents was designed to inhibit the vascular endothelial growth factor (VEGF) pathway; as VEGF is the most prevalent and dominant proangiogenic growth factor in the tumor microenvironment [7-10]. FDA approved agents that directly target the VEGF pathway include the antiVEGF antibody bevacizumab and the multitargeted tyrosine kinase inhibitors (sunitinib, sorafenib, and pazopanib), 
which inhibit VEGFRs along with other receptor tyrosine kinases [11-16].

At the present time, the factors/pathways that mediate resistance to antiangiogenic therapy are unknown. In preclinical models, there is evidence of compensatory pathways that mediate resistance at the levels of the tumor blood vessels, microenvironment, and tumor epithelial cells [17-21]. Antiangiogenic therapy may induce HIF-1 $\alpha$ and HIF- $2 \alpha$ due to tumor blood vessel constriction, decrease in blood flow, and induction of intratumoral hypoxia [22, 23]. HIF- $1 \alpha$ and HIF- $2 \alpha$, in turn, transactivate gene programs important for inducing tumor angiogenesis, cancer cell growth/survival, and energy metabolism; mediating resistance. Like many other solid tumors, overexpression of HIF- $1 \alpha$ has been shown to occur in HCC and its expression has been correlated with resistance to therapy and confers a poor prognosis [24]. This overexpression is triggered by tissue hypoxia, aberrant growth factor receptor signaling, and mutations in oncogenes and tumor suppressor genes.

\section{Clinical trials with targeted agents in HCC (Table 1)}

\section{Sorafenib}

Sorafenib is a small molecule, multi-targeted tyrosine kinase inhibitor (TKI) that mediates its antiangiogenic effect through inhibition of VEGFR-2/3 and PDGFR- $\beta$, which direct the formation of new vasculature. Sorafenib has been approved by the FDA for the treatment of hepatocellular carcinoma and renal cell cancer.

The primary controlled studies indicating the potential of single-agent sorafenib in the treatment of advanced $\mathrm{HCC}$ are the SHARP (Sorafenib HCC assessment randomized protocol) and Asian-Pacific trials. Sorafenib monotherapy in the SHARP trial-a double-blind, placebo-controlled, phase III study - proved efficacious in prolonging both median overall survival (OS) and time to radiologic progression by 3 months. The sorafenib group recorded an OS of 10.7 months as compared to 7.9 months for the placebo control [15]. Patients in the sorafenib-arm showed a median time to radiographic progression of 5.5 months while the placebo-arm was 2.8 months for the same parameter [15]. Although sorafenib was therapeutically beneficial in extending median OS and time to radiographic progression, there was no significant difference in the time to symptomatic progression between the sorafenib and placebo groups. Only $2 \%$ of the patients in the sorafenib-arm achieved a partial response (PR); without any complete responses (CR) in either the sorafenib/placebo groups. However, the disease control rate was substantially higher in the sorafenib-arm versus the control-arm (43 to $32 \%$ ) [15].

Single-agent sorafenib in the Asian-Pacific trial patients demonstrated a significant increase in median OS (6.5 months) as compared to the median OS in the control group (4.2 months). There was an increase in median time to disease progression (TTP, 2.8 months) in the sorafenib treatment group in comparison to the placebo group (TTP, 1.4 months) [25]. The clinical findings in the SHARP and Asian-Pacific studies indicate the potential therapeutic benefits of single-agent sorafenib in treating advanced HCC.

In addition to its single-agent efficacy, sorafenib has also shown a benefit when combined with doxorubicin. In a phase I study of combined sorafenib/doxorubicin, all four patients with metastatic HCC maintained stable disease state for more than 1 year of treatment [26]. In a randomized, double-blind, phase II trial, sorafenib/doxorubicin

Table 1 Single-agent and combination therapy trials in HCC

\begin{tabular}{|c|c|c|c|c|}
\hline Agent & Phase & $\mathrm{ORR} / \mathrm{PFS}^{\mathrm{a}} / \mathrm{OS}^{\mathrm{a}}$ & Comments & References \\
\hline \multirow[t]{2}{*}{ Sorafenib } & III & NS/5.5/10.7 & SHARP trial; time to radiologic progression & Llovet et al [15] \\
\hline & III & $3.3 \% / 2.8 / 6.5$ & Asian-Pacific trial; time to progression & Cheng et al. [25] \\
\hline \multirow[t]{2}{*}{ Sorafenib + Doxorubicin } & I & NS/-I- & Noted that drug combination is well tolerated & Richly et al [26] \\
\hline & II & $8 \% / 4.2 / 9.2$ & & Keating et al. [27] \\
\hline Bevacizumab & II & $13 \% / 6.9 / 12.4$ & $\begin{array}{l}\text { Serious bleeding occurred in about } 11 \% \text { of } \\
\text { patients }\end{array}$ & Siegel et al [32] \\
\hline Bevacizumab + Gemcitabine/Oxaliplatin & II & $20 \% / 5.3 / 9.6$ & GEMOX-B Trial & Zhu et al [33] \\
\hline Bevacizumab + Erlotinib & II & $25 \% / 8.9 / 15.6$ & & Thomas et al [34] \\
\hline \multirow[t]{2}{*}{ Sunitinib } & II & $\mathrm{NS} /-/-$ & $\begin{array}{l}\text { European-Asian trial; trial did not proceed to } \\
\text { second phase due to low response rate }\end{array}$ & Faivre et al [35] \\
\hline & II & $2.9 / 3.9 / 9.8$ & & Zhu et al [36] \\
\hline
\end{tabular}

ORR objective response rate, $P F S$ progression free survival, $O S$ overall survival, $N S$ no statistical significance between groups

${ }^{a}$ In months (values converted from those reported in the literature) 
combination failed to prolong median TTP; however, it prolonged median OS and progression-free survival (PFS) when compared to doxorubicin alone [27].

Sorafenib combined with transarterial chemoembolization (TACE) is currently under clinical investigation [28, 29]. It is theorized that one of the reasons for the high local recurrence rate after TACE is because of increased expression of vascular growth factors and HIF- $1 \alpha[30,31]$. A rational solution to this problem is to administer antiangiogenic agents concurrently or shortly after these procedures.

\section{Bevacizumab}

Bevacizumab is a recombinant monoclonal anti-VEGF antibody that has been approved by the FDA for the treatment of advanced breast, non-squamous non-small cell lung, and colorectal cancers when combined with chemotherapy. Bevacizumab is also approved for the treatment of renal cell cancer in combination with IFN- $\alpha$.

Siegal et al. [32] tested the efficacy of single-agent bevacizumab in metastatic HCC in a phase II trial. Fortysix patients were enrolled in this study; 12 patients received $5 \mathrm{mg} / \mathrm{kg}$ of bevacizumab and 34 patients received $10 \mathrm{mg} / \mathrm{kg}$ of bevacizumab every two weeks until disease progression. Of the 46 eligible patients, $13 \%$ had an objective response and $65 \%$ were progression-free at 6 months. Overall survival at 1,2 , and 3 years was 58,28 , and $23 \%$, respectively; and median PFS was 6.9 months. In addition, bevacizumab therapy resulted in substantially decreased tumor enhancement upon dynamic contrast-enhanced MRI and circulating VEGF concentrations. Therefore, singleagent bevacizumab has shown promise in patients with advanced HCC.

In the phase II GEMOX-B clinical trial, bevacizumab was combined with an active chemotherapy regimen (gemcitabine and oxaliplatin) in HCC [33]. In the first cycle (14 days), $10 \mathrm{mg} / \mathrm{kg}$ of bevacizumab was administered on day 1 followed by $10 \mathrm{mg} / \mathrm{kg}$ on days 1 and 15 in subsequent cycles (28 days). Gemcitabine $\left(1000 \mathrm{mg} / \mathrm{m}^{2}\right)$ and oxaliplatin $\left(85 \mathrm{mg} / \mathrm{m}^{2}\right)$ were administered on days 2 and 16. Thirty patients were assessed for efficacy in this study; $20 \%$ of the patients demonstrated an objective response, and $27 \%$ achieved stable disease (SD). Median OS was 9.6 months and median PFS was 5.3 months.

In another phase II trial, bevacizumab was also tested in combination with erlotinib [34]. Forty patients received $10 \mathrm{mg} / \mathrm{kg}$ of bevacizumab every 14 days and $150 \mathrm{mg}$ of oral erlotinib daily for 28 day cycles; anti-tumor activity was analyzed every 2 cycles via RECIST criteria. Primary end-point for this study was 16-week PFS with secondary end-points of response rate (RR) and median PFS. The 16-week PFS was $62.5 \%$ and 10 patients had a partial response (PR); median PFS was 39 weeks and the median OS was 68 weeks. Overall, results from these phase II trials suggest that bevacizumab in combination with other agents may be additive, warranting randomized trials.

\section{Sunitinib}

Sunitinib is a multi-targeted TKI that inhibit VEGFR-1/2/3, PDGFR- $\alpha / \beta$, c-kit, FLT3, and RET kinases. Sunitinib has received FDA approval for the treatment of renal cell carcinoma and gastrointestinal stromal tumors. There have been two phase II clinical trials focusing on the efficacy of sunitinib monotherapy in advanced HCC.

The European/Asian trial included 37 patients receiving $50 \mathrm{mg}$ daily sunitinib on a 4 weeks on and 2 weeks off schedule (6 week/cycle; $4 / 2$ schedule) [35]. The primary end-point and determinant of efficacy in this trial was overall RR via RECIST criteria with an expected RR of $15 \%$. However, only one patient displayed PR; therefore, outcome was well below the desired primary end-point of $15 \%$ with an actual RR of $2.7 \%$. Thirteen patients had SD over 3 months. In addition to less than expected response rates, there was severe, adverse events leading to the death of four patients from GI perforations, encephalopathy, and hepatorenal syndrome.

In another phase II study by Zhu et al. [36], 34 patients with unresectable HCC were treated with sunitinib $37.5 \mathrm{mg}$ daily for 28 days followed by 14 days of rest in 6-week cycles $(4 / 2$ schedule). The primary end-point of this trial was PFS. Even though the 3-month PFS rate failed to meet the authors' predefined targeted end point of $59 \%$, results were nonetheless encouraging showing an objective RR of $2.9 \%$, median PFS of 3.9 months and median overall survival of 9.8 months. Adverse events were modest. Grade 3 and 4 toxicities occurred in less than $20 \%$ of the patients in any particular category and most commonly included myelosuppression, fatigue and transaminase abnormalities. Moreover, analysis of response of vessel integrity to singleagent sunitinib demonstrated a drastic reduction in vascular permeability; and measurement of angiogenic plasma proteins and inflammatory cytokines showed that these biomarkers are significantly altered by sunitinib.

\section{Mechanism of resistance to antiangiogenic therapy}

Although the inhibition of tumor blood vessel formation has shown clinical efficacy, treatment resistance inevitably develops through a variety of mechanism. First, resistance can occur at the tumor endothelial cell level; evidence has shown that tumor endothelial cells may harbor unstable genomes [37]. These aberrant genomes may lead to an increase in genetic alterations and the propagation of 
mutations in subsequent endothelial cell lines. These altered cell lines may acquire resistance to molecularly targeted antiangiogenic therapy.

Another form of resistance may evolve from the redundancy of angiogenic signaling pathways; tumor dependence on a particular proangiogenic factor can be altered while being subjected to antiangiogenic agents [38]. For instance, treatment with anti-VEGF agents may result in vascular rearrangement and recruitment of excessive pericytes through the overexpression of PDGF- $\beta$. The vascular remodeling that results from pericytic over-coverage renders the neovasculature less responsive to VEGF for growth dependance effectively circumventing a blocked signaling pathway with a heavier dependence on an alternate mechanism.

Thirdly, antiangiogenic therapy may lead to the susceptibility of a tumor for metastatic spread. It has been shown that inhibition of both VEGFR and PDGFR results in an increased propensity for metastatic dissemination because the newly created hypoxic microenvironment selects for highly aggressive, invasive tumor cells [21]. Blagosklonny [39] elegantly hypothesized that antiangiogenic therapy may lead to increased intratumoral hypoxia and select for highly aggressive, metastatic tumor cells.

Tumors can also evade antiangiogenic therapy by acquiring the ability to grow without neovasculature. For instance, tumor cells may grow via a process known as "vascular cooption," which is characterized by neoplastic cells growth around pre-existing, normal tissue vasculature; these tumor cells acquire oxygen and nutrients from normal tissue blood vessels [40]. Antiangiogenic mechanisms inhibit sprouting angiogenesis which relies on the need for endothelial cell proliferation and migration. Tumor cells can evade these pruning effects via intussusception; a process characterized by the splitting of preexisting vasculature into new blood vessels without a need for VEGF expression and endothelial cell proliferation [40].

A final obstacle for antiangiogenic therapy is vasculogenic mimicry, a process by which tumor cells form bloodconducting vessels without the need for endothelial cell proliferation [41].

\section{Rationale for development of more effective antiangiogenic therapy in $\mathrm{HCC}$}

Traditional strategies to design combination therapy in clinical trials are to empirically combine agents with proven clinical efficacy. These strategies have been successful in deriving effective combination treatment. With the wealth of data available from molecular studies on tumor angiogenesis, we can now rationalize novel combinations which can be readily tested in preclinical models and then clinical trials.

Treatment with the currently available antiangiogenic agents including sunitinib, sorafenib, pazopanib, axitinib, or bevacizumab would disrupt endothelial tubes and/or pericytes through the inhibition of VEGF/VEGFR and PDGF/PDGFR signaling pathways, respectively. Pericytes are vascular smooth muscle cells that provide structural support and stabilization for sprouting endothelial tubes during vascular development. These support cells are responsible for sustaining tumor angiogenesis via paracrine production of VEGF. During tumor angiogenesis, cancer cells and endothelial cells synthesize and secrete PDGF- $\beta$, which acts in a paracrine fashion, stimulating pericytes to express VEGF. Due to their function in the propagation of tumor angiogenesis, pericytes serve as an important therapeutic target in the treatment of highly vascular cancers. Inhibiting both pericytes and endothelial cells would then lead to more significant antiangiogenic effect than inhibiting endothelial cells alone. As such, selection of clinically available agents with the most potent antiangiogenic effect would involve disrupting both the VEGF/VEGFR and PDGF/PDGFR pathways. This would include agents like sorafenib, sunitinib, pazopanib, and axitinib.

Despite maximal inhibition of VEGF/VEGFR and PDGF/PDGFR pathways, resistance reliably develops due to a process known as "reactive resistance" [39]. Effective antiangiogenic therapy works by creating a hypoxic condition devoid of nutrients for sustained cellular growth/ survival. However, intratumoral hypoxia leads to the induction of compensatory pathways that mediate resistance at the levels of the tumor blood vessels, microenvironment, and tumor epithelial cells [17, 18]. In preclinical models, the hypoxia-inducible factors, HIF- $1 \alpha$ and HIF- $2 \alpha$, have been shown to be major mediators of reactive resistance [21, 42] (Fig. 1). HIF- $1 \alpha$ and HIF- $2 \alpha$ induction would lead to the modulation of $>200$ genes important for inducing tumor angiogenesis, cancer cell growth/survival, and energy metabolism; mediating resistance [43, 44]. In addition to mediating reactive resistance, HIF- $1 \alpha$ and HIF$2 \alpha$ also mediate primary resistance due to their induction through dysregulated growth signaling by inflammatory mediators, growth factors, and mutations in cancer genes [45-54]. Like many other solid tumors, overexpression of HIF- $1 \alpha$ and HIF- $2 \alpha$ has been shown to occur in HCC and may mediate resistance to therapy [24].

Thus, combining agents that inhibit HIF- $1 \alpha$ and HIF- $2 \alpha$ with effective antiangiogenic agents, such as sorafenib, sunitinib, pazopanib, or axitinib would counteract reactive resistance and other driving forces of carcinogenesis. In combination with antiangiogenic agents, inhibiting both HIF- $1 \alpha$ and HIF- $2 \alpha$ would further disrupt tumorigenesis through two potential mechanisms. The first mechanism of 
synergy is by modulation of additional angiogenic pathways that complement those affected by the currently available antiangiogenic agents [18]. We recently reported that inhibition of HIF- $1 \alpha$ and HIF- $2 \alpha$ in cancer cells improved the antiangiogenic effect of sunitinib due to the inhibition of the proangiogenic factor, ANGPTL4, and induction of the antiangiogenic factor, TSP1 [42]. The second mechanism of synergy is through the direct effects of inhibiting HIF- $1 \alpha$ and HIF- $2 \alpha$ on tumor epithelial cell growth. HIF- $1 \alpha$ and HIF- $2 \alpha$ can be induced in cancer cells through dysregulated growth signaling by inflammatory mediators, growth factors, and mutations in cancer genes. In addition to tumor angiogenesis, HIF- $1 \alpha$ and HIF- $2 \alpha$ are responsible for a multitude of cellular processes that regulates growth/survival, invasion, and metastatic potential of tumor cells [55]. Altogether, inhibiting both HIF- $1 \alpha$ and HIF- $2 \alpha$ would effectively counteract the development of resistance to antiangiogenic therapy; since the effects of HIF inhibition would be seen in both endothelial cells and tumor cells.

To date, high-throughput small-compound screens and mechanistic studies have identified several classes of anticancer agents that disrupt HIF- $1 \alpha$ function, including inhibition of its transcriptional activity and HIF- $1 \alpha$ protein synthesis or stability [56-59]. Based on partial structural and functional similarities between HIF- $1 \alpha$ and HIF- $2 \alpha$, it is possible that many of the already identified HIF- $1 \alpha$ inhibitors would inhibit HIF- $2 \alpha$. In addition, systematic efforts are currently ongoing to identify compounds that are effective at inhibiting both HIF- $1 \alpha$ and HIF- $2 \alpha$ for

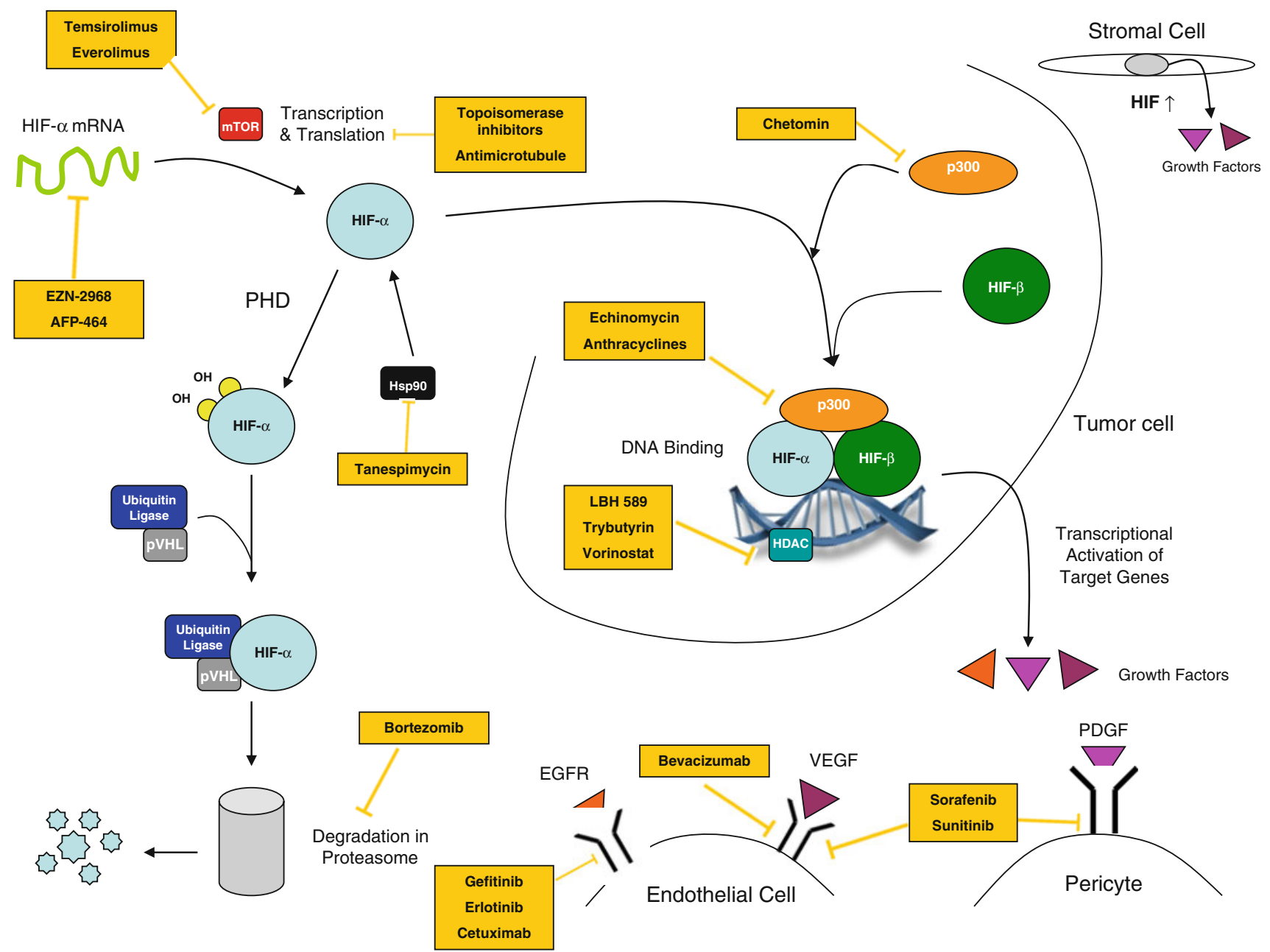

Fig. 1 Diagram depicting the tumor microenvironment and mechanism of known HIF inhibitors. HIF- $\alpha$ is overexpressed in cancer cells and stromal cells, promoting tumor angiogenesis by inducing the production of VEGF and other proangiogenic factors. HIF inhibitors work by disrupting HIF- $\alpha$ protein synthesis, stability, or transcriptional activity. $P H D$ prolyl hydroxylase, $H I F-\alpha$ hypoxia-inducible factor- $\alpha, H I F-\beta$ hypoxia-inducible factor- $\beta, p V H L$ von Hippel-Lindau protein, HDAC histone deacetylase, HSP90 heat shock protein 90 , mTOR mammalian target of rapamycin, p300 transcriptional coactivator p300, EGFR epidermal growth factor receptor, VEGF vascular endothelial growth factor, $P D G F$ platelet-derived growth factor 
Table 2 HIF inhibitors currently in clinical trials
Phase I-III status: ANR active not recruiting, $C$ completed, $E B I$ enrollment by invitation, NYR not yet recruited, $R$ recruiting, $T$ terminated, \# FDA approved

\begin{tabular}{|c|c|c|}
\hline Agent & Target & Phase/status \\
\hline AFP464 & AhR & $\mathrm{I}, \mathrm{R}$ \\
\hline Carboxyamido-triazole & $\mathrm{CCB}$ in VEGF & I, II, ANR, C \\
\hline SU5416 & c-MET & $\mathrm{I}, \mathrm{ANR}, \mathrm{C}$ \\
\hline Alvocidib (flavopiridol) & Cyclin-dependent kinase & II, C \\
\hline Decitabine & Cytosine nucleoside/DNMT & I, ANR, C, \# \\
\hline XL-647 & EGFR & $\mathrm{I}, \mathrm{C}$ \\
\hline Lonafarnib & Farnesyl-OH-transferase & II, C \\
\hline Valproic acid & HDAC & $\mathrm{I}, \mathrm{C}, \mathrm{R}, \#$ \\
\hline MS-275 & HDAC & $\mathrm{I}, \mathrm{C}$ \\
\hline Belinostat & HDAC & $\mathrm{I}, \mathrm{C}, \mathrm{R}, \#$ \\
\hline Tributyrin & HDAC & $\mathrm{I}, \mathrm{C}$ \\
\hline Trastuzumab & Her2/neu & $\mathrm{I}, \mathrm{C}, \#$ \\
\hline Vandetanib & Her2/neu; EGFR & I, II, ANR, C, R \\
\hline Romidepsin (FK228) & Histone deacetylase & II, C, \# \\
\hline Vorinostat (SAHA) & Histone deacetylase & $\mathrm{I}, \mathrm{R}, \#$ \\
\hline Panobinostat (LBH589) & Histone deacetylase & $\mathrm{I}, \mathrm{R}, \#$ \\
\hline Tanespimycin & HSP & I, II, ANR, C, EBI, NYR \\
\hline Taxoprexin & Microtubule & II, C \\
\hline Docetaxel & Microtubule & II, C, T, \# \\
\hline Ixabepilone & Microtubule & I, II, C, \# \\
\hline Patupilone & Microtubule & II, C \\
\hline XL-765 & mTOR & $\mathrm{I}, \mathrm{R}$ \\
\hline Temsirolimus & mTOR & I, II, NYR, R, \# \\
\hline Everolimus & mTOR & I, II, III, R, \# \\
\hline Pazopanib & Multitarget TKI & $\mathrm{I}, \mathrm{R}, \#$ \\
\hline Imatinib & PDGFR; cKIT & I, II, ANR, C, T, \# \\
\hline Bortezomib & Proteasome & I, II, ANR, C, \# \\
\hline EZN-2968 & RNA & $\mathrm{I}, \mathrm{R}$, \\
\hline PX-12 & Thioredoxin & I, II, C \\
\hline Topotecan & Topoisomerase & I, II, ALL, \# \\
\hline Vatalanib & VEGF & I, II, ANR, C, R \\
\hline
\end{tabular}

cancer treatment $[44,60]$. Many of these HIF inhibitors are currently in phase I and II clinical trials (Table 2 and Fig. 1).

Antiangiogenic therapy has revolutionized the treatment of HCC. Sorafenib has recently been approved for the treatment of advanced HCC; with other antiangiogenic agents, bevacizumab and sunitinib, showing promising activity. Future clinical development in HCC should include trials with HIF inhibitors, particularly in combination with antiangiogenic drugs. Appropriate doses and sequencing of these agents would need to be tested in preclinical models to help with clinical trial design.

Acknowledgments This study was funded by the National Comprehensive Cancer Network (NCCN) from general research support provided by Pfizer, Inc, NIH Grant K22CA111897, and NIH Grant R21CA115809.

Conflict of interest The authors declare no conflict of interests.
Open Access This article is distributed under the terms of the Creative Commons Attribution Noncommercial License which permits any noncommercial use, distribution, and reproduction in any medium, provided the original author(s) and source are credited.

\section{References}

1. Altekruse SF, McGlynn KA, Reichman ME (2009) Hepatocellular carcinoma incidence, mortality, and survival trends in the United States from 1975 to 2005. J Clin Oncol 27(9):1485-1491

2. Greten TF, Manns MP, Korangy F (2008) Immunotherapy of HCC. Rev Recent Clin Trials 3(1):31-39

3. Perz JF et al (2006) The contributions of hepatitis B virus and hepatitis $\mathrm{C}$ virus infections to cirrhosis and primary liver cancer worldwide. J Hepatol 45(4):529-538

4. Thomas MB, Abbruzzese JL (2005) Opportunities for targeted therapies in hepatocellular carcinoma. J Clin Oncol 23(31): 8093-8108 
5. Llovet JM, Bruix J (2008) Novel advancements in the management of hepatocellular carcinoma in 2008. J Hepatol 48(Suppl 1):S20-S37

6. Alberts SR et al (2007) Treatment options for hepatobiliary and pancreatic cancer. Mayo Clin Proc 82(5):628-637

7. Carmeliet P, Jain RK (2000) Angiogenesis in cancer and other diseases. Nature 407(6801):249-257

8. Folkman J (1995) Angiogenesis in cancer, vascular, rheumatoid and other disease. Nat Med 1(1):27-31

9. Kerbel RS (2000) Tumor angiogenesis: past, present and the near future. Carcinogenesis 21(3):505-515

10. Gale NW et al (2002) Complementary and coordinated roles of the VEGFs and angiopoietins during normal and pathologic vascular formation. Cold Spring Harb Symp Quant Biol 67:267273

11. Jain RK et al (2006) Lessons from phase III clinical trials on antiVEGF therapy for cancer. Nat Clin Pract Oncol 3(1):24-40

12. Chow LQ, Eckhardt SG (2007) Sunitinib: from rational design to clinical efficacy. J Clin Oncol 25(7):884-896

13. Hurwitz $\mathrm{H}$ et al (2004) Bevacizumab plus irinotecan, fluorouracil, and leucovorin for metastatic colorectal cancer. N Engl J Med 350(23):2335-2342

14. Bukowski RM (2004) New antiangiogenic agents for renal cell carcinoma: bevacizumab. Curr Oncol Rep 6(2):85-86

15. Llovet JM et al (2008) Sorafenib in advanced hepatocellular carcinoma. N Engl J Med 359(4):378-390

16. Sternberg CN et al (2010) Pazopanib in locally advanced or metastatic renal cell carcinoma: results of a randomized phase iii trial. J Clin Oncol 28(6):1061-1068

17. Folkman J (2004) A novel anti-vascular therapy for cancer. Cancer Biol Ther 3(3):338-339

18. Casanovas O et al (2005) Drug resistance by evasion of antiangiogenic targeting of VEGF signaling in late-stage pancreatic islet tumors. Cancer Cell 8(4):299-309

19. Mizukami $Y$ et al (2005) Induction of interleukin-8 preserves the angiogenic response in HIF-1alpha-deficient colon cancer cells. Nat Med 11(9):992-997

20. Yu JL et al (2002) Effect of p53 status on tumor response to antiangiogenic therapy. Science 295(5559):1526-1528

21. Paez-Ribes $M$ et al (2009) Antiangiogenic therapy elicits malignant progression of tumors to increased local invasion and distant metastasis. Cancer Cell 15(3):220-231

22. Yao JC et al (2008) Targeting vascular endothelial growth factor in advanced carcinoid tumor: a random assignment phase II study of depot octreotide with bevacizumab and pegylated interferon alpha-2b. J Clin Oncol 26(8):1316-1323

23. Ellis LM, Hicklin DJ (2008) VEGF-targeted therapy: mechanisms of anti-tumour activity. Nat Rev Cancer 8(8):579-591

24. Dai CX et al (2009) Hypoxia-inducible factor-1 alpha, in association with inflammation, angiogenesis and MYC, is a critical prognostic factor in patients with HCC after surgery. BMC Cancer 9:418

25. Cheng AL et al (2009) Efficacy and safety of sorafenib in patients in the Asia-Pacific region with advanced hepatocellular carcinoma: a phase III randomised, double-blind, placebo-controlled trial. Lancet Oncol 10(1):25-34

26. Richly $\mathrm{H}$ et al (2009) Combination of sorafenib and doxorubicin in patients with advanced hepatocellular carcinoma: results from a phase I extension trial. Eur J Cancer 45(4):579-587

27. Keating GM, Santoro A (2009) Sorafenib: a review of its use in advanced hepatocellular carcinoma. Drugs 69(2):223-240

28. Hoffmann K et al (2008) Prospective, randomized, double-blind, multi-center, Phase III clinical study on transarterial chemoembolization (TACE) combined with Sorafenib versus TACE plus placebo in patients with hepatocellular cancer before liver
transplantation-HeiLivCa [ISRCTN24081794]. BMC Cancer $8: 349$

29. Cabibbo G et al (2009) Multimodal approaches to the treatment of hepatocellular carcinoma. Nat Clin Pract Gastroenterol Hepatol 6(3):159-169

30. Wang B et al (2008) Increased expression of vascular endothelial growth factor in hepatocellular carcinoma after transcatheter arterial chemoembolization. Acta Radiol 49(5):523-529

31. Virmani S et al (2008) Comparison of hypoxia-inducible factor1alpha expression before and after transcatheter arterial embolization in rabbit VX2 liver tumors. J Vasc Interv Radiol 19(10): 1483-1489

32. Siegel $\mathrm{AB}$ et al (2008) Phase II trial evaluating the clinical and biologic effects of bevacizumab in unresectable hepatocellular carcinoma. J Clin Oncol 26(18):2992-2998

33. Zhu AX et al (2006) Phase II study of gemcitabine and oxaliplatin in combination with bevacizumab in patients with advanced hepatocellular carcinoma. J Clin Oncol 24(12):1898-1903

34. Thomas MB et al (2009) Phase II trial of the combination of bevacizumab and erlotinib in patients who have advanced hepatocellular carcinoma. J Clin Oncol 27(6):843-850

35. Faivre $S$ et al (2009) Safety and efficacy of sunitinib in patients with advanced hepatocellular carcinoma: an open-label, multicentre, phase II study. Lancet Oncol 10(8):794-800

36. Zhu AX et al (2009) Efficacy, safety, and potential biomarkers of sunitinib monotherapy in advanced hepatocellular carcinoma: a phase II study. J Clin Oncol 27(18):3027-3035

37. Streubel B et al (2004) Lymphoma-specific genetic aberrations in microvascular endothelial cells in B-cell lymphomas. N Engl J Med 351(3):250-259

38. Raza A, Franklin MJ, Dudek AZ (2010) Pericytes and vessel maturation during tumor angiogenesis and metastasis. Am J Hematol 85(8):593-598

39. Blagosklonny MV (2004) Antiangiogenic therapy and tumor progression. Cancer Cell 5(1):13-17

40. Rubenstein JL et al (2000) Anti-VEGF antibody treatment of glioblastoma prolongs survival but results in increased vascular cooption. Neoplasia 2(4):306-314

41. Hendrix MJ et al (2003) Vasculogenic mimicry and tumour-cell plasticity: lessons from melanoma. Nat Rev Cancer 3(6):411-421

42. Burkitt $\mathrm{K}$ et al (2009) Targeting both HIF-1 and HIF-2 in human colon cancer cells improves tumor response to sunitinib treatment. Mol Cancer Ther 8(5):1148-1156

43. Semenza GL (2003) Targeting HIF-1 for cancer therapy. Nat Rev Cancer 3(10):721-732

44. Chun SY et al (2010) Oncogenic KRAS modulates mitochondrial metabolism in human colon cancer cells by inducing HIF-1a and HIF-2a target genes. Mol Cancer 9(1):293

45. Unruh A et al (2003) The hypoxia-inducible factor-1 alpha is a negative factor for tumor therapy. Oncogene 22(21):3213-3220

46. Bos R et al (2001) Levels of hypoxia-inducible factor-1 alpha during breast carcinogenesis. J Natl Cancer Inst 93(4):309-314

47. Schindl $M$ et al (2002) Overexpression of hypoxia-inducible factor 1 alpha is associated with an unfavorable prognosis in lymph node-positive breast cancer. Clin Cancer Res 8(6): $1831-1837$

48. Koukourakis MI et al (2002) Hypoxia-inducible factor (HIF1A and HIF2A), angiogenesis, and chemoradiotherapy outcome of squamous cell head-and-neck cancer. Int J Radiat Oncol Biol Phys 53(5):1192-1202

49. Aebersold DM et al (2001) Expression of hypoxia-inducible factor1alpha: a novel predictive and prognostic parameter in the radiotherapy of oropharyngeal cancer. Cancer Res 61(7):2911-2916

50. Kurokawa $\mathrm{T}$ et al (2003) Overexpression of hypoxia-induciblefactor 1alpha (HIF-1alpha) in oesophageal squamous cell 
carcinoma correlates with lymph node metastasis and pathologic stage. Br J Cancer 89(6):1042-1047

51. Birner $\mathrm{P}$ et al (2000) Overexpression of hypoxia-inducible factor 1 alpha is a marker for an unfavorable prognosis in early-stage invasive cervical cancer. Cancer Res 60(17):4693-4696

52. Birner P et al (2001) Expression of hypoxia-inducible factor 1 alpha in epithelial ovarian tumors: its impact on prognosis and on response to chemotherapy. Clin Cancer Res 7(6):1661-1668

53. Birner P et al (2001) Expression of hypoxia-inducible factor-1 alpha in oligodendrogliomas: its impact on prognosis and on neoangiogenesis. Cancer 92(1):165-171

54. Bachtiary B et al (2003) Overexpression of hypoxia-inducible factor 1 alpha indicates diminished response to radiotherapy and unfavorable prognosis in patients receiving radical radiotherapy for cervical cancer. Clin Cancer Res 9(6):2234-2240
55. Semenza GL (2010) Defining the role of hypoxia-inducible factor 1 in cancer biology and therapeutics. Oncogene 29(5):625-634

56. Semenza GL (2009) HIF-1 inhibitors for cancer therapy: from gene expression to drug discovery. Curr Pharm Des 15(33): 3839-3843

57. Melillo G (2007) Hypoxia-inducible factor 1 inhibitors. Methods Enzymol 435:385-402

58. Escuin D, Simons JW, Giannakakou P (2004) Exploitation of the HIF axis for cancer therapy. Cancer Biol Ther 3(7):608-611

59. Giaccia A, Siim BG, Johnson RS (2003) HIF-1 as a target for drug development. Nat Rev Drug Discov 2(10):803-811

60. Scheuermann TH et al (2009) Artificial ligand binding within the HIF2 alpha PAS-B domain of the HIF2 transcription factor. Proc Natl Acad Sci U S A 106(2):450-455 